extremely low weight $(\leqslant 1,000 \mathrm{~g})$ at time of catheter insertion (OR, 9.1; $\mathrm{CI}_{95}, 1.9-42.2$ ). Catheter manipulations independently associated with CA BSI were disinfection of the catheter hub (OR, 1.2; $\left.\mathrm{CI}_{95}, 1.1-1.3\right)$, blood sampling (OR, $\left.1.4 ; \mathrm{CI}_{95}, 1.1-1.8\right)$, heparinization (OR, $\left.0.9 ; \mathrm{CI}_{95}, 0.8-1.0\right)$, and antisepsis of exit site (OR, $\left.0.9 ; \mathrm{CI}_{95}, 0.8-1.0\right)$.

This study indicates that certain manipulations (eg, blood sampling through the central line) and disconnection of the central venous catheter, which necessitates disinfection of the catheter hub, increase the risk of CA BSI, whereas other procedures (eg, heparinization and exit-site antisepsis) protect against CA BSI in neonates.

FROM: Mahieu LM, De Dooy JJ, Lenaerts AE, leven MM, De Muynck AO. Catheter manipulations and the risk of catheter-associated bloodstream infection in neonatal intensive care unit patients. J Hosp Infect 2001;48:20-26.

\section{Regional Program for Control of VRE}

In late 1996, vancomycin-resistant enterococci (VRE) were first detected in the Siouxland region of Iowa, Nebraska, and South Dakota. A task force was created, and in 1997 the assistance of the CDC was sought in assessing the prevalence of VRE in the region's facilities and implementing recommendations for screening, infection control, and education at all 32 healthcare facilities in the region.

In October 1998 and October 1999, the infection control interventions were evaluated with point-prevalence surveys and a case-control study of gastrointestinal colonization VRE, comparing infection control practices and screening policies for VRE at the acute-care and longterm-care facilities in the Siouxland region.

Perianal-swab samples were obtained from 1,954 of 2,196 eligible patients (8\%) in 1998 and 1,820 of 2,049 eligible patients $(89 \%)$ in 1999 . The overall prevalence of VRE at 30 facilities that participated in all 3 years of the study decreased from $2.2 \%$ in 1997 to $1.4 \%$ in 1998 and to $0.5 \%$ in 1999 ( $P<.001$ by chi-square test for trend). The number of facilities that had at least 1 patient with VRE declined from 15 in 1997 to 10 in 1998 to only 5 in 1999.

At both acute-care and long-term-care facilities, the risk factors for VRE colonization were prior hospitalization and treatment with antimicrobial agents. Most of the longterm-care facilities screened for vancomycin-resistant enterococci (26 of 28 in 1998 [93\%] and 23 of 25 in 1999 [92\%]) and had infection control policies to prevent the transmission of VRE (22 of 25 [88\%] in 1999). All four acutecare facilities had screening and infection control policies for VRE in 1998 and 1999.

The authors concluded that an active infection control intervention, which includes the obtaining of surveillance cultures and the isolation of infected patients, can reduce or eliminate the transmission of VRE in the healthcare facilities of a region. The authors note that the success of the intervention resulted from the collaboration among the participating health departments and the per- sonnel of the healthcare facilities and should be viewed as a model for action in this era of emerging antimicrobial resistance.

FROM: Ostrowsky BE, Trick WE, Sohn AH, Quirk $\mathrm{SB}$, Holt S, Carson LA, et al. Control of vancomycinresistant Enterococcus in health care facilities in a region. $N$ Engl J Med 2001;344:1427-1433.

\section{Guidelines for Control of West Nile Virus Infection}

The $\mathrm{CDC}$ has issued revised "Guidelines for Surveillance, Prevention, and Control of West Nile Virus Infection in the United States, April 2001." These guidelines are available from the CDC at www.cdc.gov/ncidod/dvbid and were derived from discussions during the national meeting on West Nile virus held in Charlotte, North Carolina, from January 21 to February 4, 2001. The guidelines include strategies for surveillance, laboratory diagnosis, public education, mosquito abatement, public health infrastructure changes, interjurisdictional data sharing, and research priorities.

\section{Relationship of a Clonal Outbreak of VRE to MRSA Incidence in an Australian Hospital}

Bartley and colleagues from Princess Alexandra Hospital, Brisbane, Australia, point out that Australian isolates of vancomycin-resistant enterococci (VRE) have been widely scattered geographically and are predominantly polyclonal and of the VanB phenotype. Forty-nine VRE were isolated from 47 patients in their hospital from October 1996 to December 1999; 44 of these VRE were Enterococcus faecium with a vanA glycopeptide resistance genotype. Four isolates were pathogenic. Thirty-five VRE were from an outbreak in the renal and infectious diseases units over a 4-month period.

Pulsed-field gel electrophoresis demonstrated that 41 of the 49 VRE were indistinguishable or closely related. Enhanced environmental cleaning, strict contact isolation of colonized patients, and reduced inpatient admissions terminated the epidemic. Cohorting of methicillin-resistant Staphylococcus aureus (MRSA)-positive patients was restricted, because VRE patients occupied the isolation facilities. This resulted in a statistically significant increase in MRSA infections across the hospital.

VRE epidemics have the ability to influence the epidemiology of other nosocomial pathogens when infection control resources are exhausted.

FROM: Bartley PB, Schooneveldt JM, Looke DF, Morton A, Johnson DW, Nimmo GR. The relationship of a clonal outbreak of Enterococcus faecium vanA to methicillin-resistant Staphylococcus aureus incidence in an Australian hospital. J Hosp Infect 2001;48:43-54. 